\title{
Optimizing Bioactive Substances Extraction Procedures from Guava, Olive and Potato Processing Wastes and Evaluating their Antioxidant Capacity
}

\author{
Ibrahim Khalifa ${ }^{1}$, Hassan Barakat ${ }^{1,2 *}$, Hamdy A. El-Mansy ${ }^{1}$ and Soliman A. Soliman ${ }^{1}$ \\ ${ }^{1}$ Department of Food Technology, Faculty of Agriculture, Benha University, Moshtohor, 13736 Qaliuobia, Egypt \\ ${ }^{2}$ Food Science and Human Nutrition Department, College of Agriculture and Veterinary Medicine, Qassim University, Buraidah, Kingdom of Saudi Arabia
}

"Correspondence to:

Hassan Barakat

Department of Food Technology, Faculty of

Agriculture, Benha University, Moshtohor

13736 Qaluiobia, Egypt

Tel: +201116386902

Fax: +20132467786

E-mail: hassan.barakat@fagr.bu.edu.eg

Received: November 23, 2016

Accepted: December 26, 2016

Published: December 28, 2016

Citation: Khalifa I, Barakat H, El-Mansy HA, Soliman SA. 2016. Optimizing Bioactive Substances Extraction Procedures from Guava, Olive and Potato Processing Wastes and Evaluating their Antioxidant Capacity. J Food Chem Nanotechnol 2(4): 170-177.

Copyright: (C) 2016 Khalifa et al. This is an Open Access article distributed under the terms of the Creative Commons Attribution 4.0 International License (CC-BY) (http://creativecommons. org/licenses/by/4.0/) which permits commercial use, including reproduction, adaptation, and distribution of the article provided the original author and source are credited.

Published by United Scientific Group

\begin{abstract}
Food wastes valorization has been employed dramatically at different fields due to their fine and functional components. This study is aiming at optimization of bioactive substances extraction conditions from guava, olive and potato processing wastes. After collecting, the solvent extraction technique was applied using different solvents and drying methods. Then, the bioactive substances, antioxidant capacity and antimicrobial activity were determined. Subsequently, methanol had peaked solvents either olive or potato wastes of extractable bioactive substances. Conversely, acetone was the better for both guava wastes. Hence, they also exhibited the highest scavenging activity against $\mathrm{DPPH}^{*}$ and $\mathrm{ABTS}^{*}$ free radicals. Total phenolic compounds were interrelated with antioxidant activity than other bioactive substances. Both olive wastes and guava pomace displayed greater antioxidant and antimicrobial activities than other wastes. Also, the food wastes dried using oven-drying was recommended. Formerly, it could be useful as antioxidant and antimicrobial agents in food and drug industries.
\end{abstract}

\section{Keywords}

Food processing wastes, Extraction conditions, Antioxidant activity, Bioactive substances, Antimicrobial activity

\section{Abbreviations}

AOA: Antioxidant activity; GSE: Guava seeds extract; GPE: Guava pomace extract; OLE: Olive leaves extract; OPE: Olive pomace extracts; PPRE: Potato peels russet extract; PPH: Potato peels hermus extract, TPC: Total phenolic compounds; TF: Total flavonoids

\section{Introduction}

Food processing wastes cause solicitous problems world widely. Due to its equal 39\% of total food losses [1]. Unquestionably, they occasioned from different fruits and vegetables after them converting to processed forms. However, they deemed as attractive sources of bioactive substances featured with valuable human health benefits $[2,3]$. Guava (Psidium guajava L.) wastes have upper bioactive substances than twelve tropical fruits wastes, for instance [4]. Also, they are ranged within the limits 30\% [5]. Another example, olive (Olea europaea L.) processing wastes are respectable sources for bioactive substances [6]. Especially they are remained with huge quantities after olive oil processing up to $70 \%$ of olive waste [7] and 10\% of olive leaves [8]. However, Egypt is ranked globally as the first olive in quantity per hectare to be $97.88 \mathrm{Hg} \mathrm{Ha}^{-1}$ [9]. Tangibly, potato (Solanum tuberosum L.) is the fourth largest crop grown all over the world and 
the foremost foods at more than 100 countries [10]. Whilst, potato peels (3-5\%) are the major wastes of potato processing industries [11]. It is provided an excellent source for bioactive substances [12].

Generally, polar organic solvents are the most effective in bioactive substances solubilizing from plant tissues [13]. However, there are fluctuations at previous work about optimizing conditions for bioactive substances extraction. Acetone was mentioned before as a preferring solvent [14, 15]. Whereas, methanol was recommended for bioactive substances extraction [16]. At the same time, ethanol was suggested by some author for the same purposes [17], for instance. Therefore, the present study has been undertaken with the objective of comparison between four solvents (acetone, ethanol, methanol and water) in term of bioactive substances extraction. Also, the effect of the drying methods on yield obtained, total phenolic compounds (TPC), total flavonoids (TF) and flavonols will be assessed. Moreover, their antioxidant activity (AOA) by $\mathrm{DPPH}^{\cdot}$ and $\mathrm{ABTS}^{+{ }_{+}}$assays as well as antimicrobial activity will be evaluated. Furthermore, the correlation between these components individually and their AOA using Pearson correlation and regression factor will be realized.

\section{Materials and Methods}

\section{Food processing wastes}

a. Guava seeds and pomaces were obtained from Cairo for Agricultural Processing Co., industrial zone, ElObour City, Egypt.

b. Olive leaves and pomaces Kronakii variety were obtained from Cairo for Oil Industry, industrial zone, $6^{\text {th }}$ October City, Egypt.

c. Potato peels Hermus and Russet varieties were obtained from Egypt Foods Co., industrial zone, Quesna City, Egypt.

\section{Food processing wastes preparation}

Each waste was divided into two portions after removing the unsymmetrical parties. Then it was dried by both ovendryers (Tit Axon S.R.L via Canova, Italy) at $40-50{ }^{\circ} \mathrm{C}$ gradually for $12 \mathrm{~h}$ and solar-dryers (locally made) at $\sim 38-40$ ${ }^{\circ} \mathrm{C}$ for $72 \mathrm{~h}$ till the constant weight. The dried wastes were milled (Severin, type 3871, Germany). The powder was passed through a 60 mesh sieve to obtain homogenous powder then kept at $-18 \pm 1{ }^{\circ} \mathrm{C}$ until use after packaging in dark glass jars.

\section{Bioactive substances extraction}

Experimentation, the effect of acetone, ethanol, methanol as $(80 \%)$ and water on bioactive substances extraction from each waste was inspected. Guava seeds and olive pomace were defatted by $n$-hexane as $(1: 5, \mathrm{w} / \mathrm{v})$ for $1 \mathrm{~h}$ before extraction. Each dried waste was mixed with solvent as $(1: 20, \mathrm{w} / \mathrm{v})$ individually in dark bottles. The bottles were agitated (MLM Zentrifugenbau.TS21, Germany) for $24 \mathrm{~h}$. The mixture was filtered through filter paper Whatman No.1. The filtrates were collected, then solvents were removed by rotary evaporator
(Vacuum evaporator NE-1-Rikakikai Co., LTD, Japan) at 40 ${ }^{\circ} \mathrm{C}$. They were lyophilized (CHRIST, ALPHA 1-4D plus, Germany) then kept at $-18 \pm 1{ }^{\circ} \mathrm{C}$ until further uses according to Lafka et al. [18] with some modification.

\section{Determination of total phenolic compounds}

In brief, $200 \mu \mathrm{L}$ of each extract was mixed with $1 \mathrm{~mL}$ of 10-fold diluted Folin-Ciocalteu reagent (Fluka Co., France).

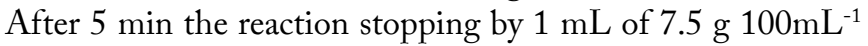
$\mathrm{Na}_{2} \mathrm{CO}_{3}$ and $1.5 \mathrm{~mL}$ distilled water was added also. The mixture was incubated in the dark for $60 \mathrm{~min}$ then the absorbance by (CE599- Automatic Scanning Spectrophotometer, GECIL, England) at $760 \mathrm{~nm}$ was measured according to Abaza et al. [19]. The TPC was expressed as milligrams of standard curve from gallic acid (Serva, fine Biochemical, New york) equivalents (mg of GAE $100 \mathrm{~g}^{-1} \mathrm{dw}$ ) using the following equation based on the calibration curve:

$\mathrm{Y}=0.0201 \mathrm{x}+0.0538$

$\left(R^{2} 0.99\right)$

Where: $\mathrm{Y}$ is the concentration and $\mathrm{x}$ is the absorbance.

\section{Determination of total flavonoids}

A $0.5 \mathrm{~mL}$ aliquot of $2 \mathrm{~g} 100 \mathrm{~mL}^{-1} \mathrm{AlCl}_{3}$ ethanolic solution was added to $0.5 \mathrm{~mL}$ of extracts and mixed well. Then, they were kept for $1 \mathrm{~h}$ at room temperature and the absorbance at $420 \mathrm{~nm}$ was measured. As for flavones, A $5.0 \mathrm{~g} 100 \mathrm{~mL}^{-1}$ sodium acetate solution were added then mixed well and kept for $2.5 \mathrm{~h}$ at room temperature. The absorbance at $440 \mathrm{~nm}$ was taken according to Mohdaly et al. [20]. The final concentration was expressed as quercetin (Sigma Aldrich, Germany) equivalents ( $\mathrm{mg} \mathrm{QEg}^{-1} \mathrm{dw}$ ) using the following equation based on the calibration curve:

$\mathrm{Y}=0.037 \mathrm{x}+0.1363$

$\left(R^{2} 0.98\right)$

Where: $\mathrm{Y}$ is the concentration and $\mathrm{x}$ is the absorbance.

\section{$\mathrm{DPPH}^{\bullet}$ radical scavenging activity}

A $0.1 \mathrm{~mL}$ from each extract and BHT $100 \mathrm{mgkg}^{-1}$ were added to $3.9 \mathrm{~mL} \mathrm{DPPH}^{*}$ (Sigma Aldrich, Germany) methanolic solution. Formerly, the absorbance at $517 \mathrm{~nm}$ was measured after the solution allowing to stand in the dark for 60 min according to Lafka et al. [18]. The final results were expressed as micromoles of Trolox equivalents per gram of dry weight ( $\left.\mu \mathrm{mol} \mathrm{TE} \mathrm{g}^{-1} \mathrm{dw}\right)$ and as $\%$ inhibition using the following equation based on the calibration curve:

$$
\operatorname{AOA}(\%)_{=}\left[\left(\mathrm{Ac}_{517}-\mathrm{As}_{517}\right) / \mathrm{Ac}_{517}\right] \times 100
$$

Where: $A c_{517}$ is the absorbance of the blank and $A s_{517}$ is the absorbance of the extracts or BHT.

\section{$\mathrm{ABTS}^{\cdot+}$ radical cation scavenging activity}

$\mathrm{ABTS}^{*+}$ radicals was produced by reacting $\mathrm{ABTS}^{-+}$ (Osaka, Japan) stock solution with $2.45 \mathrm{mmol} \mathrm{L}^{-1}$ potassium per-sulfate (final concentration) and allowing the mixture to stand in the dark at room temperature for 12-16 $\mathrm{h}$ before using. The $\mathrm{ABTS}^{*+}$ radicals' solution was diluted with ethanol to an absorbance of $0.70 \pm 0.02$ at $734 \mathrm{~nm}$ and equilibrated at $30{ }^{\circ} \mathrm{C}$ as well as measured at $734 \mathrm{~nm}$ according to $\mathrm{Lu}$ et al. [21]. The final results were expressed as $\left(\mu \mathrm{mol} \mathrm{TE} \mathrm{g}^{-1} \mathrm{dw}\right)$ and 
$\%$ inhibition using the following equation:

$\operatorname{AOA}(\%)=\left[\left(\mathrm{Ac}_{734}-\mathrm{As}_{734}\right) / \mathrm{Ac}_{734}\right] \times 100$

Where: $\mathrm{Ac}_{734}$ is control absorbance and $\mathrm{As}_{734}$ is extracts or BHT absorbance.

Evaluation of antimicrobial activity against some food pathogenic and spoilage strains

Determination of antibacterial activity

The screening of the antibacterial activity of tested extracts was performed using agar disc diffusion assay as described by Kotzekidou et al. [22]. The bacterial strains (Bacillus cereus, Escherichia coli 0157, Listeria monocytogenes, Salmonella typhi, S. typhimurium, Staphylococcus aureus and Yersinia enterocolitis) from Institut für Gärungsgewerbe, Berlin, Germany were propagated. A loop full from each strain was added into Mueller Hinton Broth (Himedia, India) then incubated at 37 ${ }^{\circ} \mathrm{C}$ for $12 \mathrm{~h}$. Appropriate volume from each culture was mixed with sterilized Mueller Hinton Agar to set an inoculums as $\sim 10^{-6}$ cell $\mathrm{mL}^{-1}$, then poured in sterilized Petri dishes. Consequently, the extracts were sterilized by $0.45 \mu \mathrm{m}$ filters (Minisart ${ }^{\circledast}$, Germany). Sterile filter paper discs $6 \mathrm{~mm}$ were immersed into sterilized extracts for $5 \mathrm{~s}$ then put immediately onto the solid cultures surface'. The plates were incubated at $37^{\circ} \mathrm{C}$ for $24-48 \mathrm{~h}$ and the inhibition zones around discs were measured.

Determination of antifungal activity

The effect of wastes extracts on mycelia yield of selected fungi were investigated at $(1,2.5$ and $5 \%)$ concentrations according to Tripathi et al. [23]. Potato dextrose broth (Himedia, India) was prepared in $50 \mathrm{~mL}$ Erlenmeyer flasks and inoculated with $10^{5}$ spore $\mathrm{mL}^{-1}$ of (Aspergillus niger, Alternaria alternata, Penicillium chrysogenum and Rbizopus stolonifer). An equal amount of distilled water was added in the corresponding control ( $0 \%$ extracts). The flasks were incubated at $28 \pm 1{ }^{\circ} \mathrm{C}$ with $120 \mathrm{rpm}$ shaking. After 5 days, flasks containing mycelia were filtered and washed through filter paper Whatman No.1. The mycelia were allowed to dry at $60^{\circ} \mathrm{C}$ for $6 \mathrm{~h}$ then at $40^{\circ} \mathrm{C}$ overnight. The growth inhibition percentage was calculated as:

$$
\text { Growth inhibition \% }=\left[\left(\mathrm{DW}_{\mathrm{utf}}-\mathrm{DW}_{\mathrm{eff}}\right) / \mathrm{DW}_{\mathrm{utf}}\right] \times 100 \ldots \text {... }
$$

Where: $\mathrm{DW}_{\text {uff }}$ - dry weight of untreated fungal strain and $\mathrm{DW}_{\text {etf }}:$ dry weight of treated fungal strain with extract.

\section{Statistical analysis}

The statistical analysis was carried out using SPSS program (ver. 19) with multi-function utility regarding to the experimental design under significance level of 0.05 for the whole results and multiple comparisons were carried out applying LSD according to Steel et al. [24]. Moreover, Pearson's correlation analysis was calculated and obtained correlation results were compared to critical values of Pearson's $r$ table under levels of significance with two-tailed test.

\section{Results and Discussions}

Effect of extraction conditions on yield obtained and total phenolic compounds of guava, olive and potato wastes extracts

Regularly, both olive wastes had peaked in their content of yield obtained and TPC. It was followed by guava, while the potato extracts had bottomed (Table 1 ). For that reason, olive leaves extract (OLE) had pointed, conversely, either potato peels russet extract (PPRE) or potato peels hermus extract (PPHE) had bottomed. Likewise, it was preceded by guava seeds extract (GSE), guava pomace extract (GPE) and olive pomace extract (OPE), respectively. Indeed, oven-drying is

\begin{tabular}{|c|c|c|c|c|c|c|c|c|c|c|}
\hline \multirow[t]{3}{*}{ Extracts } & \multirow[t]{3}{*}{ Item } & \multicolumn{8}{|c|}{ Solvent / drying methods } & \multirow{3}{*}{$\begin{array}{c}\text { Mean } \\
\pm \text { SD }\end{array}$} \\
\hline & & \multicolumn{2}{|c|}{ Acetone } & \multicolumn{2}{|c|}{ Ethanol } & \multicolumn{2}{|c|}{ Methanol } & \multicolumn{2}{|c|}{ Water } & \\
\hline & & OD & SD & OD & SD & OD & SD & OD & SD & \\
\hline \multirow{2}{*}{$\begin{array}{l}\text { Guava } \\
\text { pomace }\end{array}$} & $\mathrm{YO}^{*}$ & $120.45 \pm 1.03$ & $103.17 \pm 2.76$ & $98.79 \pm 1.19$ & $90.42 \pm 0.97$ & $107.33 \pm 0.89$ & $98.37 \pm 1.39$ & $39.95 \pm 0.25$ & $29.67 \pm 1.51$ & $86.01 \pm 31.43^{\mathrm{d}}$ \\
\hline & TPC" & $134.83 \pm 1.38$ & $99.98 \pm 2.06$ & $109.91 \pm 1.39$ & $70.59 \pm 2.17$ & $124.16 \pm 1.30$ & $80.59 \pm 1.24$ & $5.64 \pm 0.33$ & $3.51 \pm 0.32$ & $78.65 \pm 48.06^{\mathrm{c}}$ \\
\hline \multirow{2}{*}{$\begin{array}{l}\text { Guava } \\
\text { seeds }\end{array}$} & $\mathrm{YO}^{*}$ & $69.41 \pm 0.13$ & $46.55 \pm 1.02$ & $45.40 \pm 13.4$ & $36.98 \pm 1.38$ & $59.26 \pm 1.0$ & $40.35 \pm 1.21$ & $29.88 \pm 0.72$ & $18.41 \pm 0.54$ & $43.28 \pm 15.83^{c}$ \\
\hline & TPC" & $82.56 \pm 0.93$ & $66.54 \pm 0.80$ & $67.20 \pm 1.69$ & $55.35 \pm 0.77$ & $76.60 \pm 1.80$ & $61.44 \pm 1.24$ & $5.18 \pm 1.42$ & $1.57 \pm 0.21$ & $52.05 \pm 29.83^{b}$ \\
\hline \multirow{2}{*}{$\begin{array}{l}\text { Olive } \\
\text { leaves }\end{array}$} & $\mathrm{YO}^{*}$ & $261.43 \pm 0.88$ & $239.32 \pm 0.78$ & $280.33 \pm 0.92$ & $251.25 \pm 1.10$ & $299.43 \pm 3.19$ & $273.15 \pm 2.30$ & $89.38 \pm 0.81$ & $60.93 \pm 1.88$ & $219.40 \pm 87.14^{f}$ \\
\hline & TPC" & $276.04 \pm 4.56$ & $115.52 \pm 4.98$ & $349.70 \pm 17.91$ & $303.11 \pm 3.24$ & $387.08 \pm 6.68$ & $313.21 \pm 2.05$ & $99.99 \pm 3.75$ & $78.31 \pm 0.97$ & $240.37 \pm 117.49^{\mathrm{e}}$ \\
\hline \multirow{2}{*}{$\begin{array}{l}\text { Olive } \\
\text { pomace }\end{array}$} & $\mathrm{YO}^{*}$ & $167.94 \pm 1.69$ & $151.19 \pm 1.0$ & $188.04 \pm 1.39$ & $163.05 \pm 2.10$ & $200.75 \pm 3.03$ & $181.32 \pm 0.64$ & $60.11 \pm 1.55$ & $48.19 \pm 1.86$ & $145.07 \pm 55.67^{\mathrm{e}}$ \\
\hline & TPC" & $81.04 \pm 10.64$ & $67.84 \pm 1.92$ & $104.85 \pm 9.17$ & $75.48 \pm 1.76$ & $371.93 \pm 10.04$ & $198.62 \pm 10.02$ & $66.25 \pm 3.74$ & $28.70 \pm 3.73$ & $124.33 \pm 106.80^{\mathrm{d}}$ \\
\hline \multirow{2}{*}{$\begin{array}{l}\text { Potato } \\
\text { peel } \\
\text { Hermus } \\
\end{array}$} & $\mathrm{YO}^{\circ}$ & $29.98 \pm 0.55$ & $21.39 \pm 0.99$ & $35.78 \pm 1.51$ & $23.25 \pm 0.99$ & $41.06 \pm 1.62$ & $30.23 \pm 0.86$ & $13.50 \pm 3.23$ & $8.50 \pm 0.80$ & $25.46 \pm 10.57^{\mathrm{a}}$ \\
\hline & TPC"* & $4.81 \pm 0.04$ & $0.49 \pm 0.07$ & $5.13 \pm 0.14$ & $0.70 \pm 0.02$ & $9.48 \pm 0.49$ & $0.94 \pm 0.05$ & $0.94 \pm 0.48$ & $0.19 \pm 0.04$ & $2.83 \pm 3.18^{a}$ \\
\hline \multirow{2}{*}{$\begin{array}{l}\text { Potato } \\
\text { peel } \\
\text { Russet }\end{array}$} & $\mathrm{YO}^{*}$ & $34.57 \pm 2.01$ & $23.33 \pm 1.23$ & $40.19 \pm 0.91$ & $30.51 \pm 1.09$ & $46.43 \pm 1.40$ & $38.07 \pm 1.07$ & $18.37 \pm 1.31$ & $13.26 \pm 1.13$ & $30.59 \pm 10.99^{b}$ \\
\hline & TPC" & $6.73 \pm 0.44$ & $0.90 \pm 0.07$ & $7.60 \pm 0.37$ & $1.24 \pm 0.14$ & $12.00 \pm 0.58$ & $2.50 \pm 0.35$ & $0.92 \pm 0.04$ & $0.35 \pm 0.04$ & $4.03 \pm 4.07^{\mathrm{a}}$ \\
\hline
\end{tabular}


the heightened manner for yield obtained and TPC isolation than solar-drying as mentioned before for other tissues [25]. A significant difference $(p<0.05)$ among solvents was observed, and this matter is varied according to the solvent polarity [13]. Expressively, methanol had peaked (117.97 $\left.\mathrm{g} \mathrm{kg}^{-1} \mathrm{dw}\right)$. Whilst, the water was the bottommost solvent in yield obtained and TPC being 35.84 and $24.29 \mathrm{mg}$ GAE $100 \mathrm{~g}^{-1} \mathrm{dw}$, respectively. Methanol was resulted the maximum bioactive substances either olive or potato wastes. Whilst, the highest quantities from these components both guava wastes were attained using acetone as illustrated in Table 1 and Figure $1 \mathrm{~A}$. These results are agreement with Mohamed, Mohdaly and Jimenez et al. $[14,20,26]$. However, no results established about extraction optimization of these components from guava pomace.

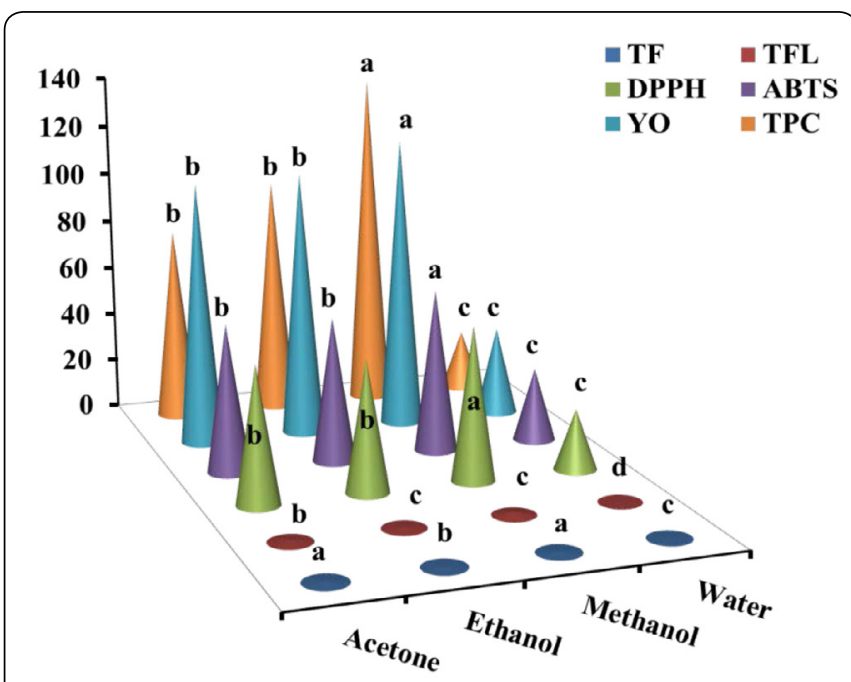

Figure 1A: Efficiency of different solvents in bioactive substances extraction from guava, potato and olive waste extract. (Mean \pm SD), $n=3$.

Determination of total flavonoids and flavonols compounds of guava, olive and potato processing wastes

Statistically, the significant differences $(p<0.05)$ between both drying methods in order of TF and flavonols contents was initiated. Equally, a significant difference $(p<0.05)$ in TF and flavonols substances was observed clearly among wastes (Table 2). Amazingly, the greater such components were taken out using acetone rather than other solvents both guava wastes. On opposite finding, olive and potato wastes methanolic extracts exhibited the highest TF and flavonols than other solvents as portrayed in Figure 1A. In the same table, water was the poorest solvent in TF and flavonols extraction except the waste kind. The OPE had pointed, whilst PPHE had bottomed from their contents of TF and flavonols. These results are in agreement with previous studies of Brahmi and Mohdaly et al. [27, 28], but are lower than published results by Abaza et al. [19]. However, no results found about such components either GPE or OPE as well extraction conditions.

Determination of antioxidant activity of guava, olive and potato processing wastes

$\mathrm{DPPH}^{*}$ radical scavenging activity

In the present work, the AOA of tested extracts was evaluated in vitro compared with BHT.Table 3 symbolized that the AOA differed significantly among solvents. Exclusively, the methanolic extracts were the highest AOA. It was monitored by acetonic and ethanolic extracts. The efficiency of AOA using different solvents was varying. For example, guava wastes acetonic extracts were the highest AOA, whereas, olive and potato wastes methanolic were the uppermost AOA compared with other solvents. Regardless drying methods or solvents, olive wastes extracts had peaked, whereas potato wastes had bottomed. Undoubtedly, oven-drying had lower influences against AOA than solar-drying a rounding 59.83 and $39.21 \%$, respectively (Figure $1 \mathrm{~B}$ ). To emphasize that, GPE exhibited greater AOA than GSE. Consequently, OLE was higher AOA than OPE. Similarly, PPRE was higher AOA than PPHE. Kui-Hua et al. [29] reported that a yellow skinned potato variety had lower AOA than the red skinned potato. The obtained data were evident that the $\mathrm{BHT}$ recorded AOA higher than GSE, PPHE and PPRE extracts and lower than OLE, OPE and GPE extracts in radical inhibition. Thus, OLE, OPE and GPE can be used a good natural AOA in food and/or stuffs. These results are agreement with the results published by Lu and Brahmi et al. [21, 27].

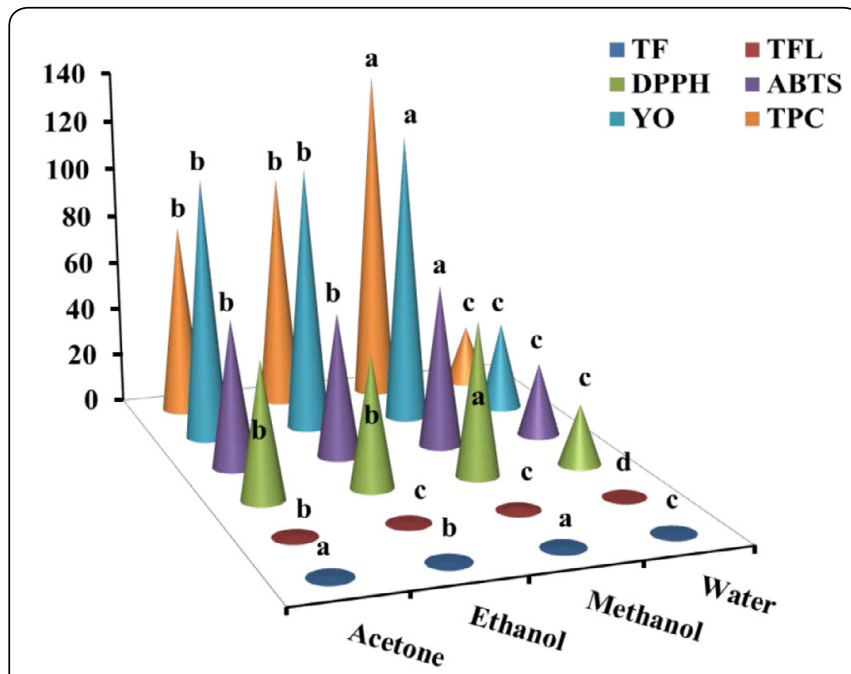

Figure 1B: Changes of bioactive substances between oven and solar-dried guava, olive and potato waste extract.(Mean $\pm \mathrm{SD}), n=3$.

\section{$\mathrm{ABTS}^{\cdot+}$ radical cation activity}

ABTS $^{*+}$ was the second method used for AOA evaluation as tabulated in Table 4 . The AOA had totally and significantly $(p<0.05)$ differed among extracts regarding solvents. Expectation the drying methods, methanol and ethanol solvent exited the highest AOA, except both guava wastes which had peaked using acetone. Conversely, the water extracts exhibited lower AOA than all wastes both drying methods. Olive wastes extracts recorded the highest extracts in AOA. Conversely, potato wastes listed the lowest extracts regardless solvents or drying methods. Also, the maximum AOA was noticed with oven-dried OLE methanolic extract's reaching 99.91\%, while the bottommost AOA was attained with solar-dried PPHE water extract's to be $5.74 \%$. The obtained data manifested that the BHT scored higher AOA than GSE, PPHE and PPRE. Whilst it was recorded lower AOA than GPE, OPE and 
Table 2: Total flavonoids and total flavonols compounds of oven- and solar-dried guava, olive and potato wastes extracts extracted using different solvents $(\mathrm{Mean} \pm \mathrm{SD}), n=3$.

\begin{tabular}{|c|c|c|c|c|c|c|c|c|c|c|}
\hline \multirow{3}{*}{ Extracts } & \multirow[t]{3}{*}{ Item } & \multicolumn{8}{|c|}{ Solvent/ drying methods } & \multirow{3}{*}{$\begin{array}{l}\text { Mean } \\
\pm \mathrm{SD}\end{array}$} \\
\hline & & \multicolumn{2}{|c|}{ Acetone } & \multicolumn{2}{|c|}{ Ethanol } & \multicolumn{2}{|c|}{ Methanol } & \multicolumn{2}{|c|}{ Water } & \\
\hline & & OD & SD & OD & SD & OD & SD & OD & SD & \\
\hline \multirow{2}{*}{$\begin{array}{l}\text { Guava } \\
\text { pomace }\end{array}$} & $\mathrm{TF}^{*}$ & $0.33 \pm 0.0$ & $0.21 \pm 0.0$ & $0.20 \pm 0.0$ & $0.08 \pm 0.0$ & $0.28 \pm 0.04$ & $0.15 \pm 0.01$ & $0.10 \pm 0.01$ & -- & $0.16 \pm 0.10^{c}$ \\
\hline & TFL" & $0.21 \pm 0.01$ & $0.19 \pm 0.01$ & $0.11 \pm 0.0$ & $0.08 \pm 0.0$ & $0.17 \pm 0.0$ & $0.14 \pm 0.0$ & $0.04 \pm 0.01$ & $0.01 \pm 0.01$ & $0.11 \pm 0.06^{c}$ \\
\hline \multirow[t]{2}{*}{ Guava seeds } & $\mathrm{TF}^{*}$ & $0.38 \pm 0.02$ & $0.26 \pm 0.02$ & $0.21 \pm 0.02$ & $0.09 \pm 0.02$ & $0.34 \pm 0.02$ & $0.22 \pm 0.02$ & $0.18 \pm 0.01$ & $0.07 \pm 0.01$ & $0.21 \pm 0.10^{\mathrm{d}}$ \\
\hline & TFL" & $0.25 \pm 0.04$ & $0.21 \pm 0.02$ & $0.13 \pm 0.01$ & $0.10 \pm 0.01$ & $0.20 \pm 0.01$ & $0.17 \pm 0.01$ & $0.03 \pm 0.0$ & -- & $0.13 \pm 0.08^{d}$ \\
\hline \multirow{2}{*}{$\begin{array}{l}\text { Olive } \\
\text { leaves }\end{array}$} & $\mathrm{TF}^{*}$ & $1.56 \pm 0.05$ & $1.11 \pm 0.05$ & $1.22 \pm 0.02$ & $0.76 \pm 0.01$ & $1.80 \pm 0.01$ & $1.34 \pm 0.02$ & $0.73 \pm 0.03$ & $0.30 \pm 0.02$ & $1.10 \pm 0.46^{\mathrm{e}}$ \\
\hline & TFL" & $2.68 \pm 0.02$ & $1.57 \pm 0.02$ & $2.54 \pm 0.01$ & $1.41 \pm 0.01$ & $4.27 \pm 0.02$ & $2.88 \pm 0.02$ & $0.45 \pm 0.02$ & $0.36 \pm 0.01$ & $2.01 \pm 1.26^{\mathrm{e}}$ \\
\hline \multirow{2}{*}{$\begin{array}{l}\text { Olive } \\
\text { pomace }\end{array}$} & $\mathrm{TF}^{*}$ & $1.79 \pm 0.02$ & $1.35 \pm 0.02$ & $1.24 \pm 0.07$ & $0.85 \pm 0.02$ & $1.88 \pm 0.06$ & $1.44 \pm 0.06$ & $0.89 \pm 0.03$ & $0.47 \pm 0.02$ & $1.23 \pm 0.46^{\mathrm{f}}$ \\
\hline & TFL" & $2.95 \pm 0.02$ & $1.85 \pm 0.01$ & $2.79 \pm 0.02$ & $1.68 \pm 0.02$ & $4.30 \pm 0.02$ & $3.20 \pm 0.02$ & $0.41 \pm 0.01$ & $0.30 \pm 0.01$ & $2.18 \pm 1.32^{\mathrm{f}}$ \\
\hline \multirow{2}{*}{$\begin{array}{l}\text { Potato peel } \\
\text { Hermus }\end{array}$} & $\mathrm{TF}^{*}$ & $0.08 \pm 0.01$ & -- & $0.18 \pm 0.01$ & $0.07 \pm 0.01$ & $0.22 \pm 0.01$ & $0.10 \pm 0.01$ & -- & -- & $0.08 \pm 0.08^{\mathrm{a}}$ \\
\hline & TFL" & $0.04 \pm 0.0$ & -- & $0.02 \pm 0.0$ & -- & $0.12 \pm 0.0$ & $0.09 \pm 0.01$ & -- & -- & $0.03 \pm 0.04^{a}$ \\
\hline \multirow{2}{*}{$\begin{array}{l}\text { Potato peel } \\
\text { Russet }\end{array}$} & $\mathrm{TF}^{*}$ & $0.18 \pm 0.01$ & $0.06 \pm 0.01$ & $0.26 \pm 0.01$ & $0.14 \pm 0.01$ & $0.29 \pm 0.01$ & $0.17 \pm 0.01$ & -- & -- & $0.13 \pm 0.10^{\mathrm{b}}$ \\
\hline & TFL" & $0.05 \pm 0.01$ & $0.02 \pm 0.01$ & $0.04 \pm 0.0$ & $0.01 \pm 0.0$ & $0.15 \pm 0.01$ & $0.11 \pm 0.01$ & $0.01 \pm 0.01$ & -- & $0.04 \pm 0.05^{b}$ \\
\hline
\end{tabular}

Total flavonoids that measured as $\mathrm{mg} \mathrm{QEg}^{-1} \mathrm{dw}$ : see materials and methods section,

" Total flavonols that measured as mg $\mathrm{QEg}^{-1} \mathrm{dw}$, OD: Oven-drying, SD: Solar drying,

$a, b, c, \ldots:$ : Means with the same letter in the same column are not significantly different $(p>0.05)$.

Table 3: Effect of different solvents and drying methods on antioxidant activity of guava, olive and potato wastes extracts (Mean \pm SD), $n=3$.

\begin{tabular}{|c|c|c|c|c|c|c|c|c|c|c|}
\hline \multirow[t]{3}{*}{ Extracts } & \multirow[t]{3}{*}{ DPPH•" } & \multicolumn{8}{|c|}{ Solvent/ drying methods } & \multirow{3}{*}{$\begin{array}{l}\text { Mean } \\
\pm \mathrm{SD}\end{array}$} \\
\hline & & \multicolumn{2}{|c|}{ Acetone } & \multicolumn{2}{|c|}{ Ethanol } & \multicolumn{2}{|c|}{ Methanol } & \multicolumn{2}{|c|}{ Water } & \\
\hline & & OD & SD & OD & SD & OD & SD & OD & SD & \\
\hline \multirow{2}{*}{$\begin{array}{l}\text { Guava } \\
\text { pomace }\end{array}$} & IP* & $90.04 \pm 1.19$ & $53.65 \pm 1.27$ & $70.95 \pm 0.72$ & $37.36 \pm 0.72$ & $79.04 \pm 0.61$ & $47.13 \pm 1.16$ & $12.43 \pm 1.19$ & $8.51 \pm 1.11$ & $49.88 \pm 28.44^{\mathrm{d}}$ \\
\hline & $\mathrm{TE}^{*+}$ & $16.24 \pm 0.26$ & $8.11 \pm 0.27$ & $12.08 \pm 0.16$ & $4.64 \pm 0.15$ & $13.84 \pm 0.13$ & $6.72 \pm 0.25$ & -- & -- & $7.70 \pm 5.81^{\mathrm{d}}$ \\
\hline \multirow{2}{*}{$\begin{array}{l}\text { Guava } \\
\text { seeds }\end{array}$} & IP* & $72.74 \pm 1.26$ & $49.26 \pm 0.98$ & $62.75 \pm 0.93$ & $19.45 \pm 1.35$ & $69.83 \pm 0.46$ & $40.75 \pm 0.84$ & $10.47 \pm 2.08$ & $1.65 \pm 0.97$ & $40.86 \pm 26.37^{\mathrm{c}}$ \\
\hline & $\mathrm{TE}^{*}$ & $12.47 \pm 0.28$ & $7.34 \pm 0.21$ & $10.29 \pm 0.20$ & $0.84 \pm 0.29$ & $11.83 \pm 0.10$ & $5.49 \pm 0.18$ & -- & -- & $6.03 \pm 5.04^{c}$ \\
\hline \multirow{2}{*}{$\begin{array}{l}\text { Olive } \\
\text { leaves }\end{array}$} & IP" & $91.58 \pm 0.92$ & $68.72 \pm 0.84$ & $97.26 \pm 0.57$ & $77.75 \pm 1.68$ & $99.36 \pm 0.56$ & $86.48 \pm 1.60$ & $67.14 \pm 0.63$ & $58.94 \pm 1.11$ & $80.90 \pm 14.39^{\mathrm{f}}$ \\
\hline & $\mathrm{TE}^{*+}$ & $16.05 \pm 0.45$ & $11.78 \pm 0.38$ & $17.93 \pm 0.13$ & $13.65 \pm 0.37$ & $18.39 \pm 0.12$ & $15.56 \pm 0.35$ & $11.32 \pm 0.14$ & $9.52 \pm 0.24$ & $14.27 \pm 3.09^{\mathrm{f}}$ \\
\hline \multirow{2}{*}{$\begin{array}{l}\text { Olive } \\
\text { pomace }\end{array}$} & IP* & $90.80 \pm 1.07$ & $64.58 \pm 1.22$ & $95.58 \pm 1.15$ & $70.39 \pm 1.15$ & $98.8 \pm 0.72$ & $83.4 \pm 1.29$ & $60.79 \pm 0.83$ & $52.39 \pm 0.83$ & $77.09 \pm 16.64^{\mathrm{e}}$ \\
\hline & $\mathrm{TE}^{*+}$ & $16.73 \pm 0.41$ & $11.49 \pm 0.50$ & $17.94 \pm 0.26$ & $12.29 \pm 0.26$ & $18.66 \pm 0.16$ & $15.21 \pm 0.29$ & $10.14 \pm 0.19$ & $8.25 \pm 0.19$ & $13.84 \pm 3.67^{\mathrm{e}}$ \\
\hline \multirow{2}{*}{$\begin{array}{l}\text { Potato } \\
\text { peel } \\
\text { Hermus }\end{array}$} & IP" & $14.87 \pm 1.07$ & $7.21 \pm 0.96$ & $20.57 \pm 0.72$ & $11.61 \pm 1.15$ & $30.34 \pm 1.16$ & $19.31 \pm 1.60$ & $5.43 \pm 0.67$ & $2.02 \pm 0.83$ & $13.92 \pm 8.94^{\mathrm{a}}$ \\
\hline & $\mathrm{TE}^{* \prime}$ & $0.57 \pm 0.08$ & -- & $1.08 \pm 0.16$ & -- & $3.20 \pm 0.25$ & $0.86 \pm 0.37$ & -- & -- & $0.714 \pm 1.05^{\mathrm{a}}$ \\
\hline \multirow{2}{*}{$\begin{array}{l}\text { Potato } \\
\text { peel } \\
\text { Russet }\end{array}$} & IP & $28.00 \pm 1.45$ & $19.73 \pm 1.04$ & $36.8 \pm 1.15$ & $26.45 \pm 0.97$ & $48.14 \pm 1.37$ & $32.47 \pm 0.72$ & $10.41 \pm 0.83$ & $2.02 \pm 0.83$ & $25.50 \pm 14.05^{\mathrm{b}}$ \\
\hline & $\mathrm{TE}^{* *}$ & $2.98 \pm 0.14$ & $0.98 \pm 0.04$ & $4.61 \pm 0.25$ & $2.52 \pm 0.23$ & $7.07 \pm 0.30$ & $3.92 \pm 0.17$ & -- & -- & $2.75 \pm 2.34^{b}$ \\
\hline \multirow[t]{2}{*}{ ВНT"- } & IP* & \multicolumn{8}{|c|}{$77.41 \pm 0.01$} & \\
\hline & $\mathrm{TE}^{* *}$ & \multicolumn{8}{|c|}{$12.77 \pm 0.02$} & \\
\hline
\end{tabular}

- Antioxidant activity was determined by DPPH' method and calculated by two ways,

Inhibitions percentage as (\%), "' Trolox equivalent $\mu \mathrm{mol} \mathrm{TE} \mathrm{g}^{-1} \mathrm{dw}$ calculated by certain equation,

- Butylated hydroxytoluene (100 ppm), OD: Oven-drying, SD: Solar drying, -- Not detected,

a,b, c, $\ldots$ : Means with the same letter in the same column are not significantly different $(p>0.05)$.

OLE around $82.49 \%$. These results are in agreement with the results published by Brahmi et al. [27]. However, until now there are no previous studies to estimate the AOA for guava wastes using $\mathrm{ABTS}^{++}$method.
Pearson correlation coefficient between bioactive substances of guava, olive and potato wastes extracts and their antioxidant activity

Obviously, the correlation between yield obtained and TPC was higher than its correlation with TF or flavonols 
Table 4: Antioxidant activity of oven- and solar-dried guava, olive and potato wastes extracts measured using ABTS ${ }^{+}$method $($Mean $\pm S D), n=3$.

\begin{tabular}{|c|c|c|c|c|c|c|c|c|c|c|}
\hline \multirow[t]{3}{*}{ Extracts } & \multirow[t]{3}{*}{ ABTS ++ } & \multicolumn{8}{|c|}{ Solvent/ drying methods } & \multirow{3}{*}{$\begin{array}{c}\text { Mean } \\
\pm S D\end{array}$} \\
\hline & & \multicolumn{2}{|c|}{ Acetone } & \multicolumn{2}{|c|}{ Ethanol } & \multicolumn{2}{|c|}{ Methanol } & \multicolumn{2}{|c|}{ Water } & \\
\hline & & OD & SD & OD & SD & OD & SD & OD & SD & \\
\hline \multirow{2}{*}{$\begin{array}{l}\text { Guava } \\
\text { pomace }\end{array}$} & IP* & $95.43 \pm 0.62$ & $58.07 \pm 1.29$ & $73.90 \pm 0.79$ & $43.15 \pm 0.98$ & $84.08 \pm 0.29$ & $51.56 \pm 0.83$ & $16.24 \pm 0.28$ & $14.2 \pm 1.05$ & $54.57 \pm 28.36^{\mathrm{d}}$ \\
\hline & $\mathrm{TE}^{\prime \prime}$ & $17.42 \pm 0.14$ & $9.27 \pm 0.28$ & $12.72 \pm 0.18$ & $6.01 \pm 0.22$ & $14.94 \pm 0.06$ & $7.85 \pm 0.18$ & $0.14 \pm 0.06$ & -- & $8.54 \pm 6.13^{\mathrm{d}}$ \\
\hline \multirow{2}{*}{$\begin{array}{l}\text { Guava } \\
\text { seeds }\end{array}$} & $\mathrm{IP}^{*}$ & $74.36 \pm 0.49$ & $56.81 \pm 0.95$ & $70.37 \pm 0.95$ & $29.22 \pm 0.41$ & $72.86 \pm 0.14$ & $44.86 \pm 0.87$ & $17.01 \pm 1.96$ & $6.78 \pm 0.72$ & $46.53 \pm 25.28^{c}$ \\
\hline & $\mathrm{TE}^{* \prime}$ & $12.82 \pm 0.11$ & $8.99 \pm 0.21$ & $11.95 \pm 0.21$ & $2.97 \pm 0.09$ & $12.49 \pm 0.03$ & $6.39 \pm 0.19$ & $0.31 \pm 0.43$ & -- & $6.98 \pm 5.14^{c}$ \\
\hline \multirow{2}{*}{$\begin{array}{l}\text { Olive } \\
\text { leaves }\end{array}$} & $\mathrm{IP}^{*}$ & $96.97 \pm 0.34$ & $71.42 \pm 1.49$ & $98.46 \pm 0.44$ & $83.86 \pm 0.49$ & $99.91 \pm 0.08$ & $88.33 \pm 1.16$ & $71.42 \pm 1.49$ & $65.26 \pm 1.16$ & $84.45 \pm 13.12^{\mathrm{f}}$ \\
\hline & $\mathrm{TE}^{* \prime}$ & $17.75 \pm 0.08$ & $12.18 \pm 0.33$ & $18.08 \pm 0.10$ & $14.89 \pm 0.11$ & $18.39 \pm 0.02$ & $15.87 \pm 0.26$ & $12.18 \pm 0.33$ & $10.84 \pm 0.25$ & $15.02 \pm 2.86^{\mathrm{f}}$ \\
\hline \multirow{2}{*}{$\begin{array}{l}\text { Olive } \\
\text { pomace }\end{array}$} & $\mathrm{IP}^{*}$ & $93.44 \pm 0.88$ & $71.05 \pm 0.77$ & $98.37 \pm 0.36$ & $74.13 \pm 0.82$ & $99.09 \pm 0.21$ & $87.47 \pm 0.93$ & $66.53 \pm 1.16$ & $61.2 \pm 0.75$ & $81.41 \pm 14.33^{\mathrm{e}}$ \\
\hline & $\mathrm{TE}^{\mathrm{m}}$ & $16.98 \pm 0.19$ & $12.10 \pm 0.17$ & $18.06 \pm 0.08$ & $12.77 \pm 0.18$ & $18.22 \pm 0.05$ & $15.68 \pm 0.20$ & $11.11 \pm 0.25$ & $9.95 \pm 0.16$ & $14.35 \pm 3.12^{\mathrm{e}}$ \\
\hline \multirow{2}{*}{$\begin{array}{l}\text { Potato } \\
\text { peel } \\
\text { Hermus }\end{array}$} & $\mathrm{IP}^{*}$ & $20.44 \pm 1.02$ & $12.30 \pm 1.70$ & $29.49 \pm 0.82$ & $17.28 \pm 0.82$ & $35.37 \pm 1.02$ & $22.03 \pm 0.68$ & $8.23 \pm 1.02$ & $5.74 \pm 0.68$ & $18.86 \pm 9.78^{a}$ \\
\hline & $\mathrm{TE}^{* \prime}$ & $1.06 \pm 0.22$ & -- & $3.03 \pm 0.18$ & $0.36 \pm 0.18$ & $4.31 \pm 0.22$ & $1.40 \pm 0.15$ & -- & -- & $1.27 \pm 1.54^{\mathrm{a}}$ \\
\hline \multirow{2}{*}{$\begin{array}{l}\text { Potato } \\
\text { peel } \\
\text { Russet }\end{array}$} & $\mathrm{IP}^{*}$ & $34.06 \pm 0.75$ & $23.88 \pm 1.38$ & $43.19 \pm 1.63$ & $32.11 \pm 0.75$ & $57.53 \pm 2.18$ & $39.35 \pm 1.03$ & $15.24 \pm 0.89$ & $6.38 \pm 0.55$ & $31.46 \pm 15.51^{b}$ \\
\hline & $\mathrm{TE}^{* \prime}$ & $4.03 \pm 0.16$ & $1.81 \pm 0.3$ & $6.02 \pm 0.36$ & $3.60 \pm 0.17$ & $9.15 \pm 0.47$ & $5.31 \pm 0.23$ & $0.04 \pm 0.07$ & -- & $3.74 \pm 2.99^{\mathrm{b}}$ \\
\hline \multirow[t]{2}{*}{ ВНT"' } & IP* & \multicolumn{8}{|c|}{$82.49 \pm 0.14$} & \\
\hline & $\mathrm{TE}^{* \prime}$ & \multicolumn{8}{|c|}{$13.81 \pm 0.01$} & \\
\hline
\end{tabular}

- Antioxidant activity was determined by $\mathrm{ABTS}^{\cdot+}$ method and calculated by two ways,

"Inhibitions percentage as (\%), "' Trolox equivalent $\mu \mathrm{mol} \mathrm{TE} \mathrm{g}^{-1} \mathrm{dw}$ calculated by certain equation,

- Butylated hydroxytoluene (100 ppm), OD: Oven-drying, SD: Solar drying, -- Not detected,

$a, b, c, \ldots:$ Means with the same letter in the same column are not significantly different $(p>0.05)$.

as presented in Table 5. This may be due to the difference of chemical composition between phenolic and flavonoids extractability [30]. Also the correlation between yield obtained and $\mathrm{AOA}$ against $\mathrm{DPPH}^{*}$ or $\mathrm{ABTS}^{-+}$had pointed compared with (TPC,TF, flavonols) and AOA. Due to the yield obtained included TPC, TF and flavonols but collectively. Surely, the highest correlation was observed between $\mathrm{DPPH}^{\circ}$ and $\mathrm{ABTS}^{++}$ due to the mechanism are similar as mentioned before [31]. From the previous data, TPC was the major components in yield obtained. Thus, the regression between TPC and AOA towards $\mathrm{DPPH}^{\cdot}$ and $\mathrm{ABTS}^{++}$assay was presented in Figure 2. A high content of TPC led to high AOA in each extract. For example, AOA was posted the highest incidence to be $84.45 \%$ when TPC rating $240.37 \mathrm{mg}$ GAE $100 \mathrm{~g}^{-1} \mathrm{dw}$. In contrast the ratio of their decreased to the lowest levels when the TPC was decreased.

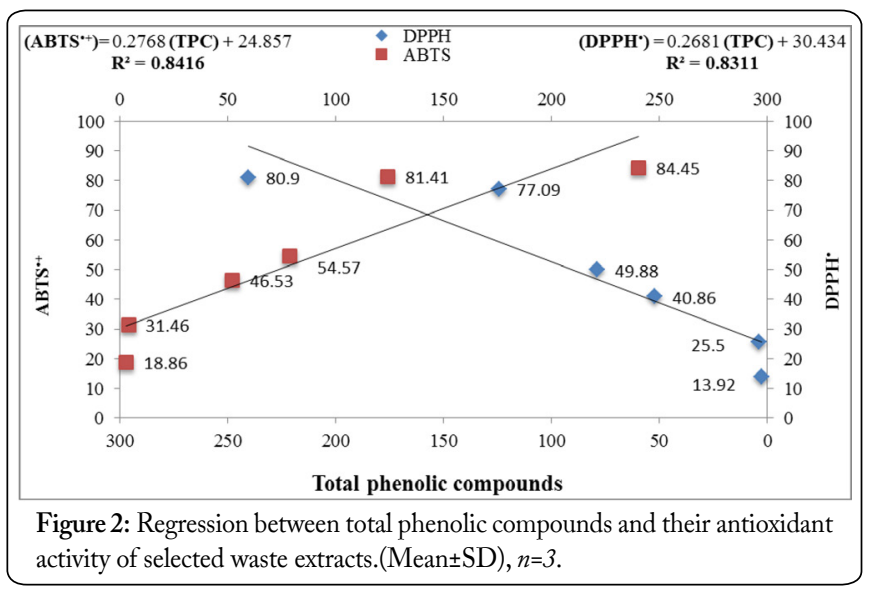

Table 5: Pearson's correlation coefficients of bioactive substances and their antioxidant activity in guava, olive and potato extracts.

\begin{tabular}{|c|c|c|c|c|c|c|}
\hline \multicolumn{7}{|c|}{ Bioactive substances } \\
\hline & $\begin{array}{c}\text { YO } \\
\left(\mathrm{g} \mathrm{kg}^{-1}\right. \\
\mathrm{dw})\end{array}$ & $\begin{array}{c}\text { TPC } \\
\left(\mathrm{mg} \mathrm{g}^{-1}\right. \\
\mathrm{dw})\end{array}$ & $\begin{array}{c}\text { TF } \\
(\mathrm{mg} \\
\mathrm{QE} \mathbf{g}^{-1} \\
\text { dw) }\end{array}$ & $\begin{array}{l}\text { TFL } \\
(\mathrm{mg} \\
\mathrm{QE} \mathrm{g}^{-1} \\
\mathrm{dw})\end{array}$ & $\begin{array}{l}\text { DPPH' } \\
(\mu \mathrm{mol} \\
\mathrm{TE} \mathrm{g}^{-1} \\
\mathbf{d w})\end{array}$ & $\begin{array}{c}\text { ABTS }^{\cdot+} \\
(\mu \mathrm{molTE} \\
\left.\mathrm{g}^{-1} \mathbf{d w}\right)\end{array}$ \\
\hline $\mathrm{YO}$ & 1 & 0.92 & $0.87^{+\cdots}$ & $0.89^{-\cdots}$ & 0.82 & $0.81^{--}$ \\
\hline TPC & & 1 & $0.80^{-\infty}$ & $0.84^{-*+}$ & $0.78^{-\cdots+}$ & $0.76^{--}$ \\
\hline TF & & & 1 & $0.95^{\ldots+*}$ & $0.76^{+*+*}$ & $0.75^{+\infty}$ \\
\hline TFL & & & & 1 & $0.70^{n+m}$ & $0.68^{\prime \prime \prime}$ \\
\hline $\mathrm{DPPH}^{\circ}$ & & & & & 1 & $0.99^{*-\infty}$ \\
\hline $\mathrm{ABTS}^{++}$ & & & & & & 1 \\
\hline
\end{tabular}

Asterisks $(* *, * *)$ represent a significant difference at $(P<0.01, P<0.001)$, respectively.

Evaluation of the antimicrobial activities for guava, olive and potato wastes extracts

Antibacterial activity

According the aforementioned results, the best extracts for each waste was preferred. Then, its antibacterial activity in vitro against seven bacterial strains was studied; data were summarized in Table 6. Noticeably, OLE exhibited the highest antibacterial activity in different levels. It was followed by OPE and GPE. In contract, PPRE and GSE showed the lowest impacts against the same pathogenic strains. OLE was greater significantly $(p<0.05)$ than OPE. Whilst, no significant differences $(p>0.05)$ were evident between OPE and GPE. 
Table 6: Effect of guava, olive and potato wastes extracts on the growth inhibition of some food pathogenic bacterial strains $(\mathrm{Mean} \pm \mathrm{SD}), n=3$.

\begin{tabular}{|c|c|c|c|c|c|c|c|}
\hline \multirow[t]{2}{*}{ Extracts" } & \multicolumn{7}{|c|}{ Strains / Inhibition zone (mm) } \\
\hline & 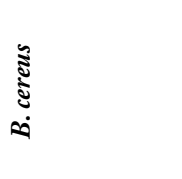 & $\begin{array}{l}10 \\
0 \\
0 \\
0 \\
0 \\
0 \\
0\end{array}$ & 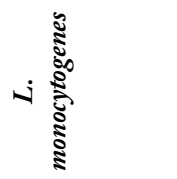 & $\underset{\dot{s}}{\vec{j}}$ & is & 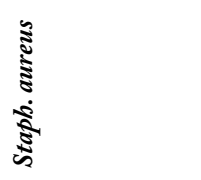 & 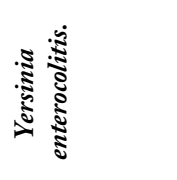 \\
\hline GPE & $10.00 \pm 0.0^{\mathrm{cA}}$ & $9.50 \pm 0.71^{\mathrm{bB}}$ & $9.00 \pm 0.0^{\mathrm{aB}}$ & $10.50 \pm 0.71^{\mathrm{dB}}$ & $9.50 \pm 0.71^{\mathrm{bB}}$ & $10.00 \pm 0.0^{\mathrm{cB}}$ & $10.00 \pm 0.0^{c \mathrm{C}}$ \\
\hline GSE & $11.00 \pm 0.0^{\mathrm{dB}}$ & $7.50 \pm 0.71^{\mathrm{aA}}$ & $8.50 \pm 0.71^{\mathrm{bA}}$ & $12.00 \pm 0.0^{\mathrm{eC}}$ & $7.50 \pm 0.71^{\mathrm{aA}}$ & $10.00 \pm 0.0^{\mathrm{cB}}$ & $12.00 \pm 0.0^{\mathrm{eD}}$ \\
\hline OLE & $11.00 \pm 0.0^{\mathrm{aB}}$ & $15.50 \pm 0.71^{\mathrm{dE}}$ & $17.00 \pm 5.66^{\mathrm{eE}}$ & $20.50 \pm 0.71^{\mathrm{gE}}$ & $14.00 \pm 1.41^{\mathrm{cD}}$ & $13.00 \pm 0.0^{\mathrm{bC}}$ & $20.00 \pm 0.0^{\mathrm{fF}}$ \\
\hline OPE & $12.00 \pm 0.0^{\mathrm{bC}}$ & $11.00 \pm 0.0^{\mathrm{aD}}$ & $25.00 \pm 0.0^{\mathrm{gF}}$ & $16.00 \pm 0.0^{\mathrm{eD}}$ & $13.00 \pm 1.41^{\mathrm{cC}}$ & $20.50 \pm 0.71^{\mathrm{fD}}$ & $15.00 \pm 0.0^{\mathrm{dE}}$ \\
\hline PPHE & $12.50 \pm 0.71^{\mathrm{cD}}$ & $10.00 \pm 0.0^{\mathrm{bC}}$ & $13.00 \pm 0.0^{\mathrm{dD}}$ & $10.00 \pm 0.0^{\mathrm{bA}}$ & -- & $13.00 \pm 0.0^{\mathrm{dC}}$ & $7.50 \pm 0.71^{\mathrm{aA}}$ \\
\hline PPRE & $10.00 \pm 0.0^{\mathrm{cA}}$ & $10.00 \pm 0.0^{\mathrm{cC}}$ & $10.00 \pm 0.0^{\mathrm{cC}}$ & $10.50 \pm 0.71^{\mathrm{dB}}$ & - & $8.50 \pm 0.71^{\mathrm{bA}}$ & $8.00 \pm 0.0^{\mathrm{aB}}$ \\
\hline
\end{tabular}

Generally, the highest inhibition against E. coli O157, S. typhi, S. typhimurium and Yersinia enterocolitis was achieved by OLE. Specifically, OPE displayed the highest activity against $L$. monocytogenes approximately $25.00 \mathrm{~mm}$. PPHE and PPRE didn't effect on S. typhimurium. Nevertheless, PPHE showed the uppermost effect against B. cereus to be 12.50 $\mathrm{mm}$. the phenolic compounds could have an inhibiting effect on microbial growth according to their constitutions and concentrations $[32,33]$. However, few studies were reported the potential antibacterial of GPE, GSE and OEL $[17,34]$. Unfortunately, no available data about antibacterial of both potatoes peel and OPE were established.

\section{Antifungal activity}

Normally, OPE and OLE was upper inhibition than other extracts. It was trailed by GPE and GSE as rendered in Figure 3. Regardless the fungi strains species, the mean value of OPE and OLE inhibition at 5\% was around (22.20 and $18.87 \%)$, respectively. Alternatively, the same concentration of PPHE had bottomed against fungal growth to be $3.72 \%$. A significant difference $(p<0.05)$ was found between each extract (2.5 and 5\%) once. GPE affected the tested fungal strains higher than GSE in different concentrations. GSE and GPE had peaked (5\%) against $A$. alternata about (13.10\% and $15.05 \%$ inhibition), respectively. OLE (5\%) was subdued significantly $(p<0.05)$ fungal strains growth than OPE with the equal concentration. To the best of our knowledge, there is no publication was found about this issue.

\section{Conclusion}

A successful and innovative comparison between four familiar solvents and tow drying methods were carried out. Methanol was the best solvents in olive and potato processing wastes according to their content of yield obtained, TPC, TF, flavonols and AOA. Conversely, acetone was the best solvents for both guava wastes, while water recorded lower bioactive substances and AOA than all solvents among extracts. Also,
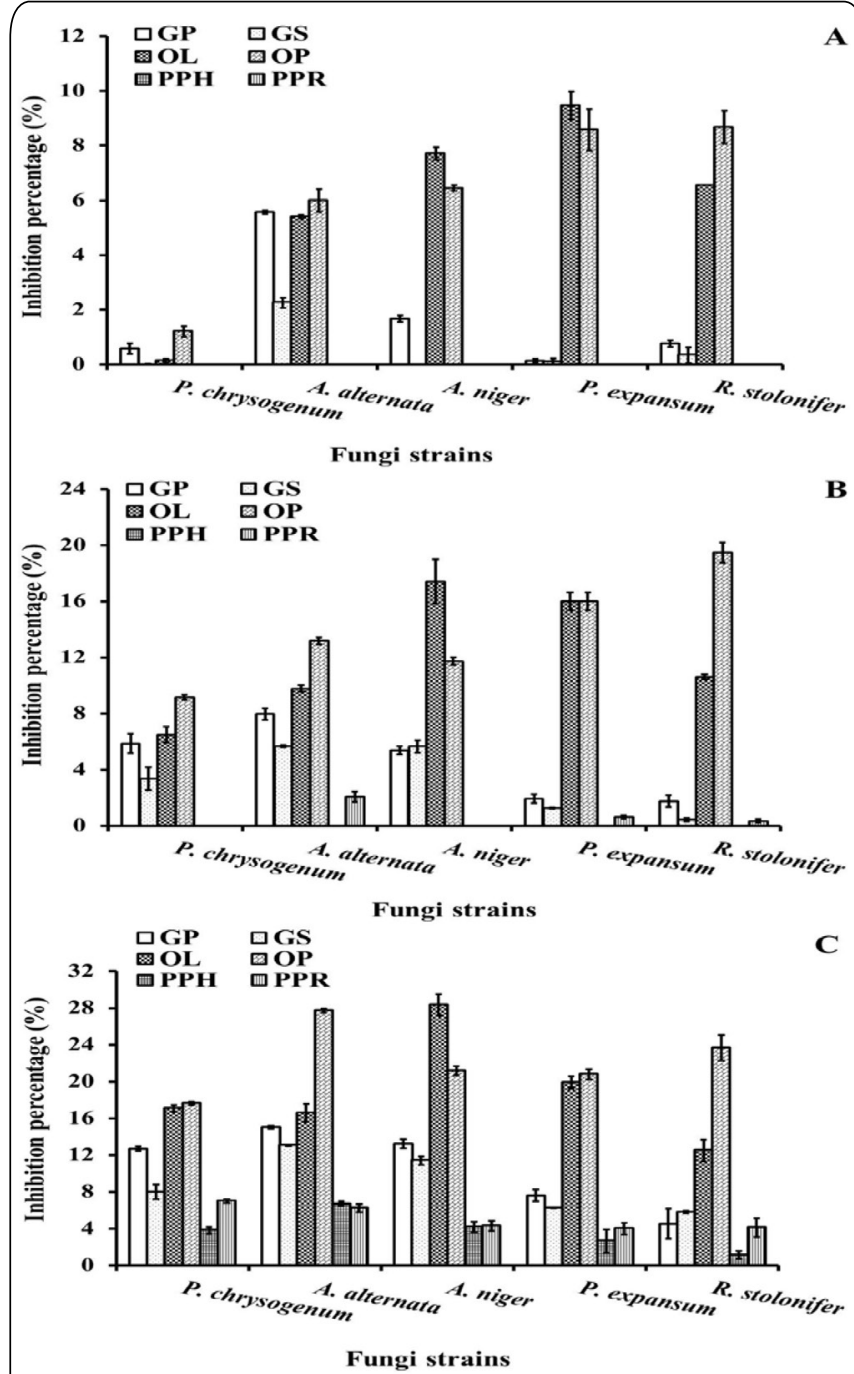

Figure 3: Evaluation of antifungal activity for guava, olive and potato wastes extracts against some spoilage fungal strains at different concentrations $1 \%$ (A), $2.5 \%$ (B) and $5 \%(\mathrm{C})$. (Mean $\pm \mathrm{SD}), n=3$. 
oven-drying led to obtain a high content of these components compared to solar-drying. Noteworthy, OLE, OPE and GPE may be used as antioxidants agent rather than BHT when they extracts were taken using the recommended conditions.

\section{Acknowledgment}

The authors gratefully acknowledge the financial support from the Benha university project [Utilization of some agricultural wastes in food and feed processing - A/1/2] 2013-2015.

\section{References}

1. European Commission. 2010. Preparatory study on food waste across EU 27, Technical Report, pp 010-054.

2. Roselló-Soto E, Galanakis CM, Brnčić M, Orlien V, Trujillo FJ, et al. 2015. Clean recovery of antioxidant compounds from plant foods, by-products and algae assisted by ultrasounds processing. Modeling approaches to optimize processing conditions. Trends Food Sci Tech 42(2): 134-149. doi: 10.1016/j.tifs.2015.01.002.

3. El-Shourbagy GA, El-Zahar KM. 2014. Oxidative stability of ghee as affected by natural antioxidants extracted from food processing wastes. Ann of Agric Sci 59(2): 213-220. doi: 10.1016/j.aoas.2014.11.008.

4. Silva LMR, Figueiredo EAT, Ricardo NMPS, Vieira IGP, Figueiredo RW, et al. 2014. Quantification of bioactive compounds in pulps and by-products of tropical fruits from Brazil. Food Chem 143: 398-404. doi: 10.1016/j.foodchem.2013.08.001.

5. Jiménez-Escrig A, Rincón M, Pulido R, Saura-Calixto F. 2001. Guava fruit (Psidium guajava $\mathrm{L}$.) as a new source of antioxidant dietary fiber. $J$ Agr Food Chem 49(11): 5489-5493. doi: 10.1021/jf010147p.

6. Terpinc P, Čeh B, Ulrih NP, Abramovič H. 2012. Studies of the correlation between antioxidant properties and the total phenolic content of different oil cake extracts. Ind Crop Pro 39: 210-217. doi: 10.1016/j.indcrop.2012.02.023

7. Sánchez MP, Ruiz MV. 2006. Production of pomace olive oil. Grasas Yaceites 57(1): 47-55.

8. Bouaziz M, Fki I, Jemai H, Ayadi M, Sayadi S. 2008. Effect of storage on refined and husk olive oils composition: stabilization by addition of natural antioxidants from Chemlali olive leaves. Food Chem 108(1): 253-262. doi: 10.1016/j.foodchem.2007.10.074.

9. FAO, Production of oilcrops primary.

10. Leo L, Leone A, Longo C, Lombardi DA, Raimo F, et al. 2008. Antioxidant compounds and antioxidant activity in "early potatoes". $J$ Agr Food Chem 56(11): 4154-4163. doi: 10.1021/jf073322w.

11. Dhingra D, Michael M, Rajput H. 2012. Physico-chemical characteristics of dietary fibre from potato peel and its effect on organoleptic characteristics of biscuits. J Agr Eng 49(4): 25-32.

12. Amado IR, Franco D, Sánchez M, Zapata C, Vázquez JA. 2014. Optimisation of antioxidant extraction from Solanum tuberosum potato peel waste by surface response methodology. Food Chem 165: 290-299. doi: 10.1016/j.foodchem.2014.05.103.

13. Kim JH, Chang SM, Kim I, Kim YJ, Hwang J, et al. 2007. Design of optimal solvent for extraction of bio-active ingredients from mulberry leaves. Biochem EngJ 37(3): 271-278. doi: 10.1016/j.bej.2007.05.006.

14. Mohamed GF,Mohamed SS, Taha FS.2011. Antioxidant, antimicrobial, and anticarcinogenic properties of Egyptian guava seed extracts. Nature and Science 9(11): 32-41.

15. Sun T, Xu Z, Godber JS, Prinyawiwatkul W. 2006. Capabilities of oat extracts in inhibiting cholesterol and long chain fatty acid oxidation during heating. Cereal Chem J83(4): 451-454. doi: 10.1094/cc-83-0451.

16. Peschel W, Sánchez F, Diekmann W, Plescher A, Gartzía I, et al. 2006. An industrial approach in the search of natural antioxidants from vegetable and fruit wastes. Food Chem 97(1): 137-150. doi: 10.1016/j. foodchem.2005.03.033.
17. Guilherme PJ, Porto E, Bani C, Matias de alencar S, Micotti da gloria $\mathrm{E}$, et al. 2012. Antimicrobial potential and chemical composition of agro-industrial wastes. J Nat Prod 5: 27-36.

18. Lafka T, Lazou AE, Sinanoglou VJ, Lazos ES. 2011. Phenolic and antioxidant potential of olive oil mill wastes. Food Chem 125(1): 92-98. doi: 10.1016/j.foodchem.2010.08.041.

19. Abaza L, Ben Youssef N, Manai H, Haddada FM, Methenni K, et al. 2011. Chétoui olive leaf extracts: influence of the solvent type on phenolics and antioxidant activities. Grasas y aceites 62(1): 96-104. doi: 10.3989/gya.044710

20. Mohdaly AAA, Hassanien MFR, Mahmoud A, Sarhan MA, Smetanska I. 2013. Phenolics extracted from potato, sugar beet, and sesame processing by-products. Int J Food Prop 16(5): 1148-1168.

21. Lu J, Zhao H, Chen J, Fan W, Dong J, et al. 2007. Evolution of phenolic compounds and antioxidant activity during malting. $\mathrm{J} \mathrm{Agr} \mathrm{Food} \mathrm{Chem}$ 55(26): 10994-11001. doi: 10.1021/jf0722710.

22. Kotzekidou P, Giannakidis P, Boulamatsis A. 2008. Antimicrobial activity of some plant extracts and essential oils against foodborne pathogens in vitro and on the fate of inoculated pathogens in chocolate. LWT - Food Sci Technol 41(1): 119-127. doi: 10.1016/j.1wt.2007.01.016.

23. Tripathi A, Sharma N, Sharma V. 2009. In vitro efficacy of Hyptis suaveolens L. (Poit.) essential oil on growth and morphogenesis of Fusarium oxysporum f.sp. gladioli (Massey) Snyder and Hansen. World J Microbiol Biotechnol 25(3): 503-512. doi: 10.1007/s11274-008-9916-y.

24. Steel R, Torrie J, Dickey D. 1997. Principles and procedures of statistics: a biometrical approach. 3rd ed, McGraw-Hill, New York, NY, USA

25. Al-Weshahy A, El-Nokety M, Bakhete M, Rao V. 2013. Effect of storage on antioxidant activity of freeze-dried potato peels. Food Res Int 50(2): 507-512. doi: 10.1016/j.foodres.2010.12.014.

26. Jimenez P, Masson L, Barriga A, Chávez J, Robert P. 2011. Oxidative stability of oils containing olive leaf extracts obtained by pressure, supercritical and solvent-extraction. Eur J Lipid Sci Technol 113(4): 497505. doi: 10.1002/ejlt.201000445.

27. Brahmi F, Mechri, B, Dabbou, S, Dhibi, M, and Hammami, M. 2012. The efficacy of phenolics compounds with different polarities as antioxidants from olive leaves depending on seasonal variations. Ind Crop Prod 38: 146-152. doi: 10.1016/j.indcrop.2012.01.023.

28. Mohdaly AA, Hassanien MFR, Mahmoud A, Sarhan MA, Smetanska I. 2012. Phenolics extracted from potato, sugar beet, and sesame processing by-products. Int $J$ Food Prop 16(5): 1148-1168. doi: 10.1080/10942912.2011.578318.

29. Kui-Hua L, Eung-Jun P, Hoi-Seon L, Dong-Man K, Stephen LL, et al. 2006. Evaluation of potato varieties with high antioxidant activities by measuring phenolic acids in different tuber parts. Hort Environ Biotechnol 47(3): 126-131.

30. Floegel A, Kim D, Chung S, Koo SI, Chun OK. 2011. Comparison of ABTS/DPPH assays to measure antioxidant capacity in popular antioxidant-rich US foods. J Food Compos Anal 24(7): 1043-1048. doi: 10.1016/j.jfca.2011.01.008.

31. Chen Y, Wang J, Ou Y, Chen H, Xiao S, et al. 2014. Cellular antioxidant activities of polyphenols isolated from Eucalyptus leaves (Eucalyptus grandis x Eucalyptus urophylla GL9). J Func Foods 7: 737-745. doi: 10.1016/j.jff.2013.12.003.

32. Alberto MR, Farías ME, Manca de Nadra MC. 2001. Effect of gallic acid and catechin on Lactobacillus hilgardii $5 w$ growth and metabolism of organic compounds. J Agr Food Chem 49(9): 4359-4363. doi: 10.1021/ jf0101915.

33. Rodríguez VMJ, Tomassini SLR, Manca de nadra MC, Strasser de saad AM. 2010. Antioxidant capacity and antibacterial activity of phenolic compounds from argentinean herbs infusions. Food Cont 21(5): 779785. doi: 10.1016/j.foodcont.2009.10.017.

34. Mohamed GF,Mohamed SS,Taha FS.2011. Antioxidant, antimicrobial, and anticarcinogenic properties of Egyptian guava seed extracts. Nature and Science 9(11): 32-41. 